\title{
Reply to the letter by Fujishiro et al. concerning "Controversy regarding gastric cancer and diabetes"
}

\author{
Akira Sekikawa $^{1} \cdot$ Hirokazu Fukui $^{2} \cdot$ Takanori Maruo $^{1} \cdot$ Takehiko Tsumura $^{1}$. \\ Yukio Osaki $^{1}$
}

Received: 7 August 2015/Accepted: 9 August 2015/Published online: 29 August 2015

(C) Springer Japan 2015

We thank Fujishiro et al. for their interest in our recently published article [1] and for their insightful comments. Several reports have focused on the association between diabetes mellitus (DM) and the risk of gastric cancer [2,3]. In previous studies, however, the criteria for both DM and the diagnosis of gastric cancer varied, which means that any results suggesting an association between DM and gastric cancer development remain controversial. Our endoscopic follow-up study clearly showed that the presence of DM diagnosed on the basis of medical history and/or serological examination was associated with the development of early gastric cancer [4].

We compared the clinical features of patients with gastric xanthelasma between those who developed early gastric cancer and those who did not. Interestingly, there was a significantly higher incidence of DM and a higher level of fasting plasma glucose in the gastric xanthelasma patients who developed gastric cancer than in those who did not (46.7 vs. $19.6 \%, p=0.043$ and $120.3 \pm 11.6$ vs. $105.2 \pm 2.2, \quad p=0.043$, respectively). Gastric cancer development showed no significant relationship to age, sex, body mass index or the level of hemoglobin A1c. Unfortunately, we did not obtain any data on plasma insulin and c-peptide, and the number of patients who developed early gastric cancer was small. However, our study demonstrated

This reply refers to the article available at doi: 10.1007/s00535-015-1113-9.

Akira Sekikawa

sekky@osaka-med.jrc.or.jp

1 Department of Gastroenterology and Hepatology, Osaka Red Cross Hospital, 5-30, Fudegasaki, Tennoji-ku, Osaka 543-8555, Japan

2 Division of Gastroenterology, Department of Internal Medicine, Hyogo College of Medicine, Nishinomiya, Japan unequivocally that the presence of DM was significantly related to the development of early gastric cancer in patients with gastric xanthelasma.

Naturally, it is very important to examine the presence of Helicobacter pylori infection and to evaluate the severity of gastric atrophy in order to predict the development of early gastric cancer in patients undergoing endoscopic examination [1,5]. However, our data additionally demonstrate that xanthelasma may also be a useful predictive marker for the development of early gastric cancer. Endoscopic examination is very helpful for evaluation of gastric atrophy and xanthelasma, and therefore endoscopic surveillance may be advisable to isolate patients who are at risk of gastric cancer development at an earlier stage.

Compliance with ethical standards

Conflict of interest The authors have declared no conflicts of interest.

\section{References}

1. Seikawa A, Fukui H, Sada R, et al. Gastric atrophy and xanthelasma are marker for predicting the development of gastric cancer. J Gastroenterol. 2015; d doi:10.1007/s00535-015-1081-0.

2. Ikeda F, Doi Y, Yonemoto K, et al. Hyperglycemia increases risk of gastric cancer posed by Helicobacter pylori infection: a population-based cohort study. Gastroenterology. 2009; 136:1234-41.

3. Shimoyama S. Diabetes mellitus carries a risk of gastric cancer: a meta-analysis. World J Gastroenterol. 2013;19:6902-10.

4. Sekikawa A, Fukui H, Maruo T, et al. Diabetes mellitus increases the risk of early gastric cancer development. Eur $\mathrm{J}$ Cancer. 2014;50:2065-71.

5. Uemura N, Okamoto S, Yamamoto S, et al. Helicobacter pylori infection and the development of gastric cancer. N Engl J Med. 2001;345:784-9. 